\title{
On Securing a Door with Finger Print Biometric Technique
}

\author{
Adeolu Afolabi, Oke Alice \\ Department of Computer Science and Engineering \\ Ladoke Akintola University of Technology, Ogbomoso. Nigeria \\ adefolabius@yahoo.com
}

\begin{abstract}
Security of valuables is as paramount as their acquisition. Valuables ranging from human lives to expensive resources and sensitive data need to be tightly secured. In this present day when armed robbery has gotten more sophisticated particularly in developing countries, there is the need for tighter security means, and one of the most secured technologies that can be employed is biometrics, finger print door lock to be precise. Biometrics is the science and technology of measuring and analysing biological data, biometrics measures and analyses human body characteristics such as DNA, fingerprint, eye retina and iris, voice pattern, facial pattern and hand measurement [1].

The software that drives the microcontroller was done using the $C$ language on MPLAB compiler, the coding was segmented into various modules; first, the module that drives the LCD screen, next is the module that drives the finger print scanner, this instructs the scanner to first register users and allow the inputted finger print to be compared with the pre-registered finger prints. The pre-registered finger prints are saved on the IC registers of the Micro controller. An alert is sounded whenever a fingerprint that is not found in the Microcontroller memory is placed on the scanner as an intruder.

The construction of this project was done in three different stages, the writing of the code (driver) which controls the Microcontroller using $C$ language, the implementation of the whole project on a solder-less experiment board, the soldering of the circuits on Vero-boards and the coupling of the entire project to the casing. The implementation of this project was done on the breadboard as a prototype, the power supply was first derived from a bench power supply in the electronics laboratory, in all the development guaranteed security for illegal intrusion of illegal entity to room, the mechanism can be implemented in a broader sense on a door where a there is restriction of access.
\end{abstract}




\section{INTRODUCTION}

The design of security door lock using the finger print technology was built around a microcontroller, PIC16F628A, which reads in finger prints from finger print scanner and grant access, to a protected room, only to pre-registered finger print(s).

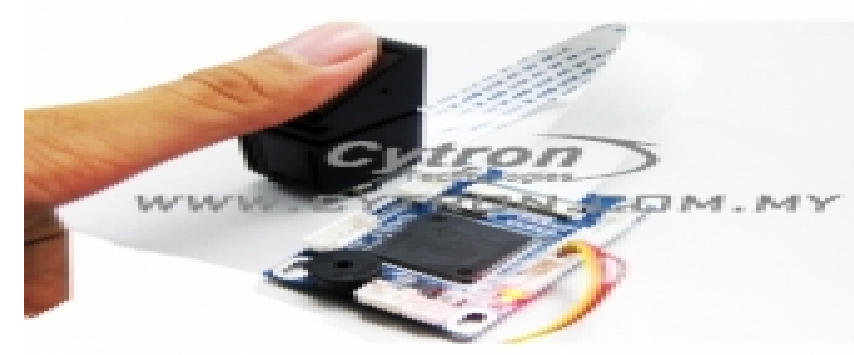

Figure 1 The picture of the SN-FPR-UART finger print reader and PIC16F628A Microcontroller.

The finger print scanner serves as the main input into this security system. Finger prints read are compared to those ones pre-programmed into the memory of the microcontroller. When a match is made, the microcontroller outputs a $\mathrm{HIGH}$ which activates the transistor-relay switching stage that controls opening and closing of the modelled motorized door granting access into the protected building.

A 16x2 alphanumeric liquid crystal display is used in this design to show the operating status of this embedded security system. By default it displays a welcome message requesting that the user should enter a finger print. And when a match is made it displays a corresponding message. The stages that make up the design of the development of a finger print control using standard door finger print scanner are explained fully in the third chapter of this project report.

\section{PRINCIPLE OF OPERATION}

The design of security door lock using the finger print technology was built around a MicroController Unit (MCU), PIC16F628A, which reads in finger prints from finger print scanner and grant access, to a protected compartment, only to pre-registered finger prints. The finger print scanner serves as the main input into this embedded security system. Finger prints read are compared to those ones pre-programmed into the memory of the microcontroller. When a match is made, the microcontroller outputs a HIGH which activates the transistor-relay switching stage that controls opening and closing of the modelled motorized door granting access into the protected building.

An alphanumeric liquid crystal display (LCD) is used in this design to show the operating status of this embedded security system. By default it displays a welcome message requesting that the user should enter a finger print. And when a match is made it displays "ACCESS GRANTED" otherwise it displays "ACCESS DENIED". 


\subsection{Hardware Design and Configuration}

The hardware design of a security door using fingerprint is achieved by interfacing a SNFPR-UART Finger reader with the PIC16F648A Microcontroller, which is the heart of the design, it is the brain box that controls the whole security door system. An LCD status display is employed to show the operating status of the system. A door movement mechanism is used in the design to make the automated door system move in clockwise and anti-clockwise directions.

\subsubsection{SN-FPR-UART Fingerprint Reader}

The finger print input stage was implemented using the SN-FPR-UART. It is a compact fingerprint reader module which is specially designed for project development because of its robustness and the following outstanding features:

a) Small in size, clear imaging, fast acquisition, high identification speed, able to sense on dry or wet fingers, supports wide range of applications.

b) High stability, convenience (direct access to any MCU through Serial UART (Universal Asynchronous Receiver/Transmitter) operation and integrated with low current warming.

c) Able to retrieve fingerprint raw data and fingerprint identity files.

d) Using commercial algorithms with high recognition speed and the collection of fingerprint done by a gentle touch on the sensing area.

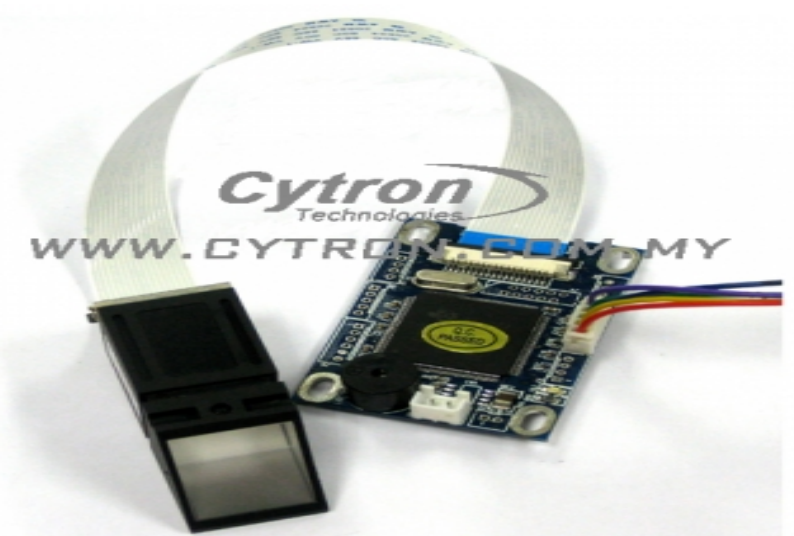

Figure 2 The picture of the SN-FPR-UART Finger Print Reader from Cytron Inc.

\subsubsection{THE PIC16F648A}

The PIC16F648A is the Microcontroller Unit used in this project work. The PIC16F628A belongs to a class of 8-bit Microcontrollers of RISC architecture. It is an 18 pin dual in-line package chip. The PIC16F628A is a tiny but complete computer. It has a CPU (central processing unit), program memory (PROM), working memory (RAM), and two input-ports. The CPU is the "brain" of the computer. It reads and executes instructions from the program memory. As it does so, it 
can store and retrieve data in working memory (RAM). CPUs make a distinction between "registers" located within the CPU and "RAM" outside it; the PIC does not, and its generalpurpose working RAM is also known as registers." On the 'F628A, there are 68 bytes of generalpurpose RAM, located at addresses $C$ to hex $4 \mathrm{~F}$. Besides the general-purpose memory, there is a special "working register" or register" where the CPU holds the data it is working on. There are also several special function registers each of which controls the operation of the PIC in some way. The program memory of the 'F648A consists of flash EPROM; it can be recorded and erased electrically, and it retains its contents when powered off. Program memory (FLASH) - for storing a written program. Since memory made in FLASH technology can be programmed and cleared more than once, it makes this microcontroller suitable for device development. EEPROM - data memory that needs to be saved when there is no supply. It is usually used for storing important data that must not be lost if power supply suddenly stops. Many other PICs require ultraviolet light for erasure and are not erasable if you buy the cheaper version without the quartz window. The chip, however, is always erasable and reprogrammable.

\subsubsection{The LCD Status Display}

The operating status display stage was implemented using the HD44780 based 16x2 LCD which is cheap and can display characters. Figure 3.3 shows the picture of the LCD used with the pin description. The Alphanumeric Liquid Crystal Display (LCD) is used in this design to show the operating status of this embedded security system. By default it displays a welcome message requesting that the user should enter a finger print. And when a match is made it displays "ACCESS GRANTED" otherwise it displays "ACCESS DENIED”.

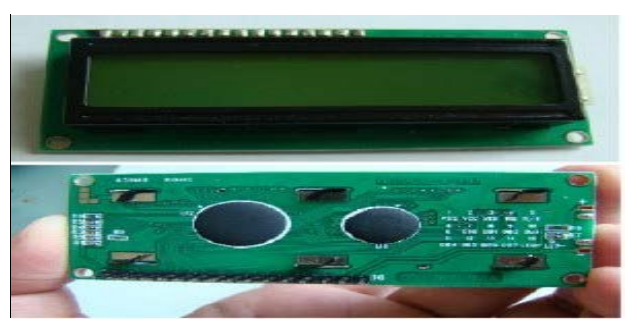

Figure.3 HD44780 based 16x2 LCD

\subsection{The Door Movement Mechanism}

The mechanism of the motor drive for the modelled automated door is built around a 5V d.c motor controlled in both directions (clockwise and anti-clockwise). To make a d.c motor go in both directions, the polarity of the d.c motor is interchanged.

Transistorised relay switch stages are needed because the outputs of the Microcontroller cannot source enough current to drive the motor directly. When a fingerprint is matched with any of the pre stored fingerprints, the Microcontroller outputs a high (digital 1) on the A1 output pin to drive the motor in the clockwise direction, thereby opening the automated door. 
The high output on $A 1$ is given a duration of ten seconds while output A2 remains low (digital 0).

After the ten seconds duration of high on A1, the Microcontroller outputs a high of A2 and a low on $\mathrm{A} 1$ for the duration of five seconds, to drive the motor in the anti-clockwise direction, thereby closing the door.

The whole control of the motor direction is implemented by toggling the A1 and A2 according to the table below

Table.1: the truth table for the control of the motor from the outputs of the MCU

\begin{tabular}{|c|c|c|}
\hline \multicolumn{2}{|c|}{ Outputs } & Motor response \\
\hline A1 & A2 & Off \\
\hline 0 & 0 & Anticlockwise \\
\hline 0 & 1 & Clockwise \\
\hline 1 & 0 & . \\
\hline
\end{tabular}

The door itself was fabricated using flat ply-wood sheets. Adhesive gum was used to join the wooden sheets together to form the body of the door system

\subsection{Software Design}

The software that drives the microcontroller was done using the Clanguage on MPLAB compiler from Microchip Technology Inc. The coding was segmented into various modules; first, the module that drives the LCD screen, this instructs the LCD to display status messages and other messages like 'Place Finger'; next is the module that drives the finger print scanner, this instructs the scanner to first register users and allow the inputted finger print to be compared with the pre-registered finger prints. The pre-registered finger prints are saved on the IC registers of the Micro controller. An alert is sounded whenever a fingerprint that is not found in the Microcontroller memory is placed on the scanner as an intruder.

\section{DATA FLOW DIAGRAM}

This shows the flow of data between different segments of the security door system as shown below: 


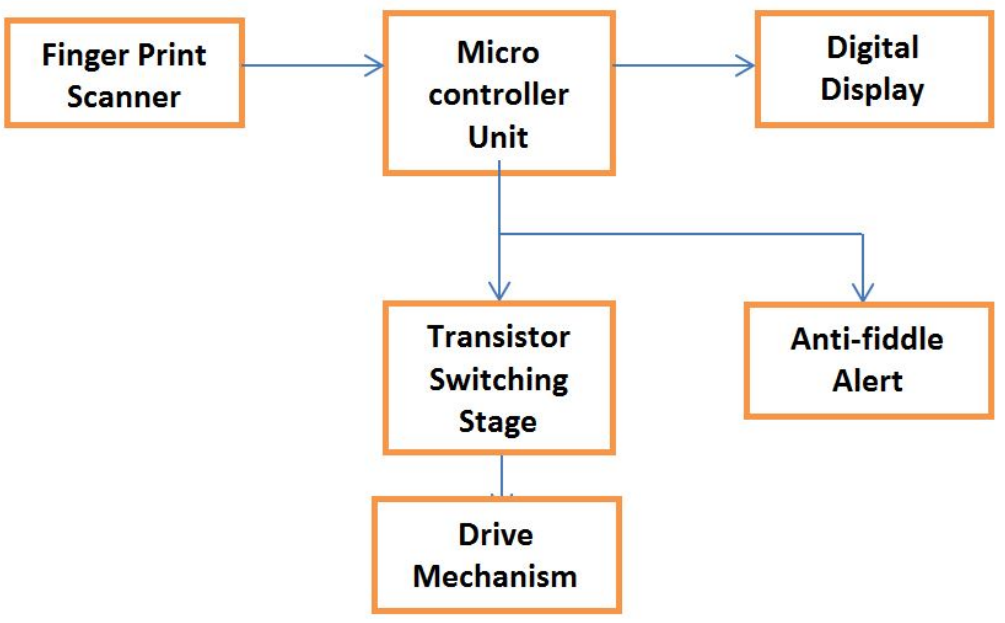

Figure 4: Data flow diagram

\section{CONSTRUCTION AND TESTING}

\subsection{Construction}

The physical realization of the project is very vital. Here the paper work is transformed into a finished hardware. After carrying out all the paper design and analysis, the project was implemented, constructed and tested to ensure its working ability. The construction of this project was done in three different stages.

1) The writing of the code (driver) which controls the Microcontroller using $C$ language (MPLAB Compiler of Microchip Technology Inc.)

2) The implementation of the whole project on a solder-less experiment board.

3) The soldering of the circuits on Vero-boards.

4) The coupling of the entire project to the casing.

\subsection{Implementation}

The implementation of this project was done on the breadboard. The power supply was first derived from a bench power supply in the electronics laboratory. To confirm the workability of the circuits before the power supply stage was soldered. The implementation of the project on bread board was successful and it met the desired design aims with each stage performing as designed.

\section{Simulation}

The whole electrical part of the project was simulated on PROTEUS simulation platform before the soldering work commenced to observe the operation of the whole project.

Figures 4.1 a-d shows the simulation screen shots 


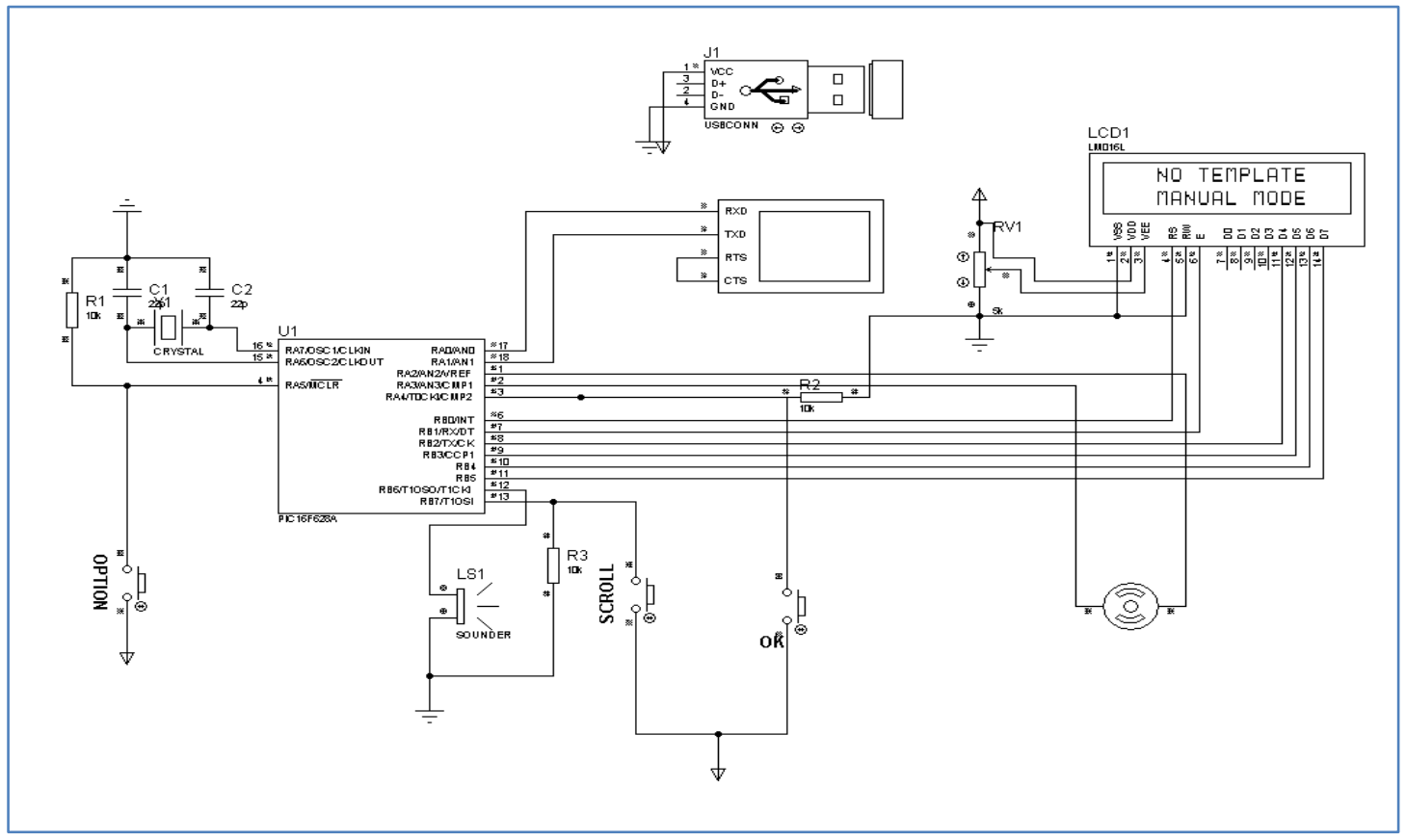

Figures 4.1a shows the simulation screen shot before any finger print was stored

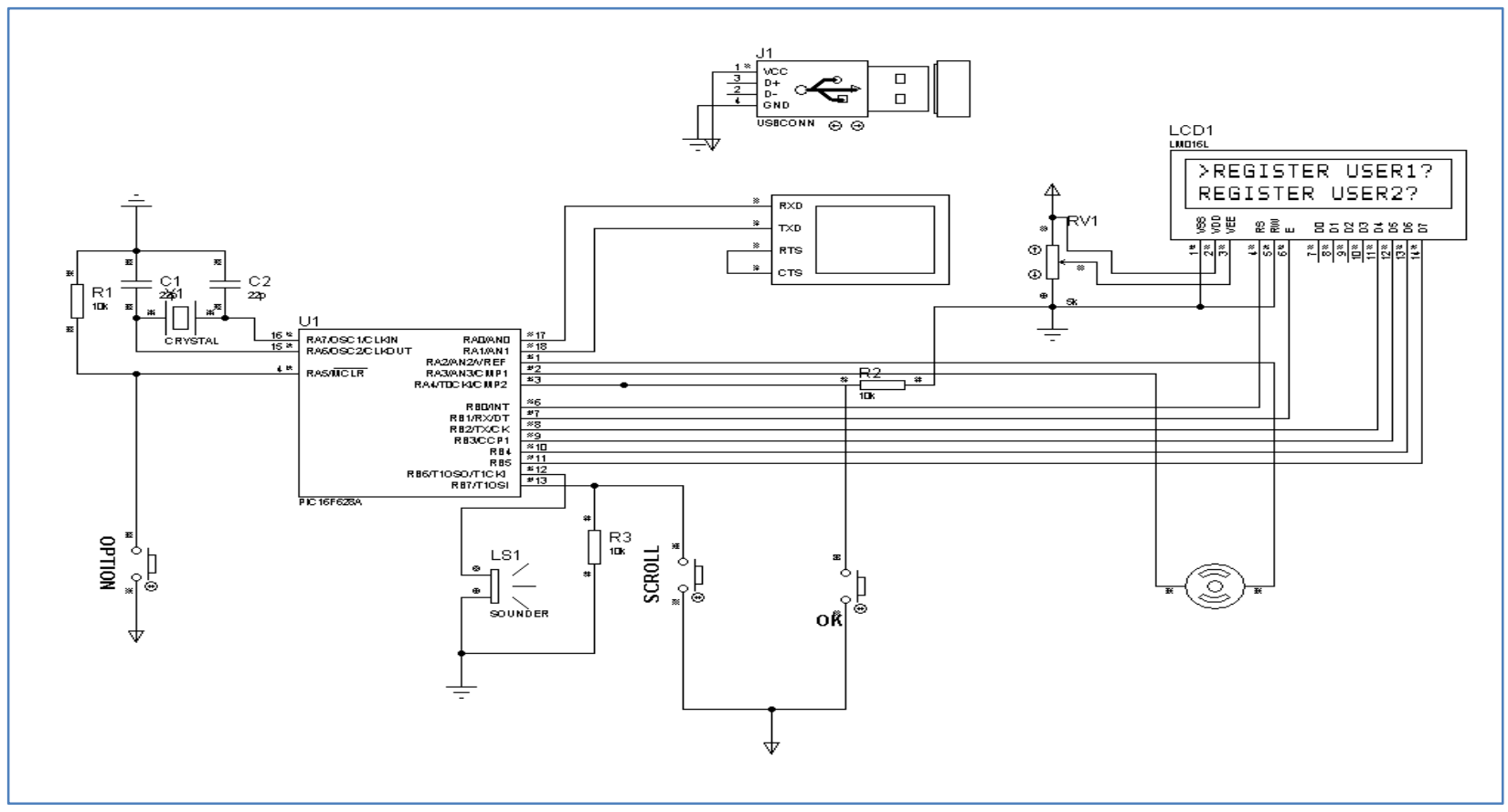

Figures $4.1 \mathrm{~b}$ shows the simulation screen shot at registering finger print. 


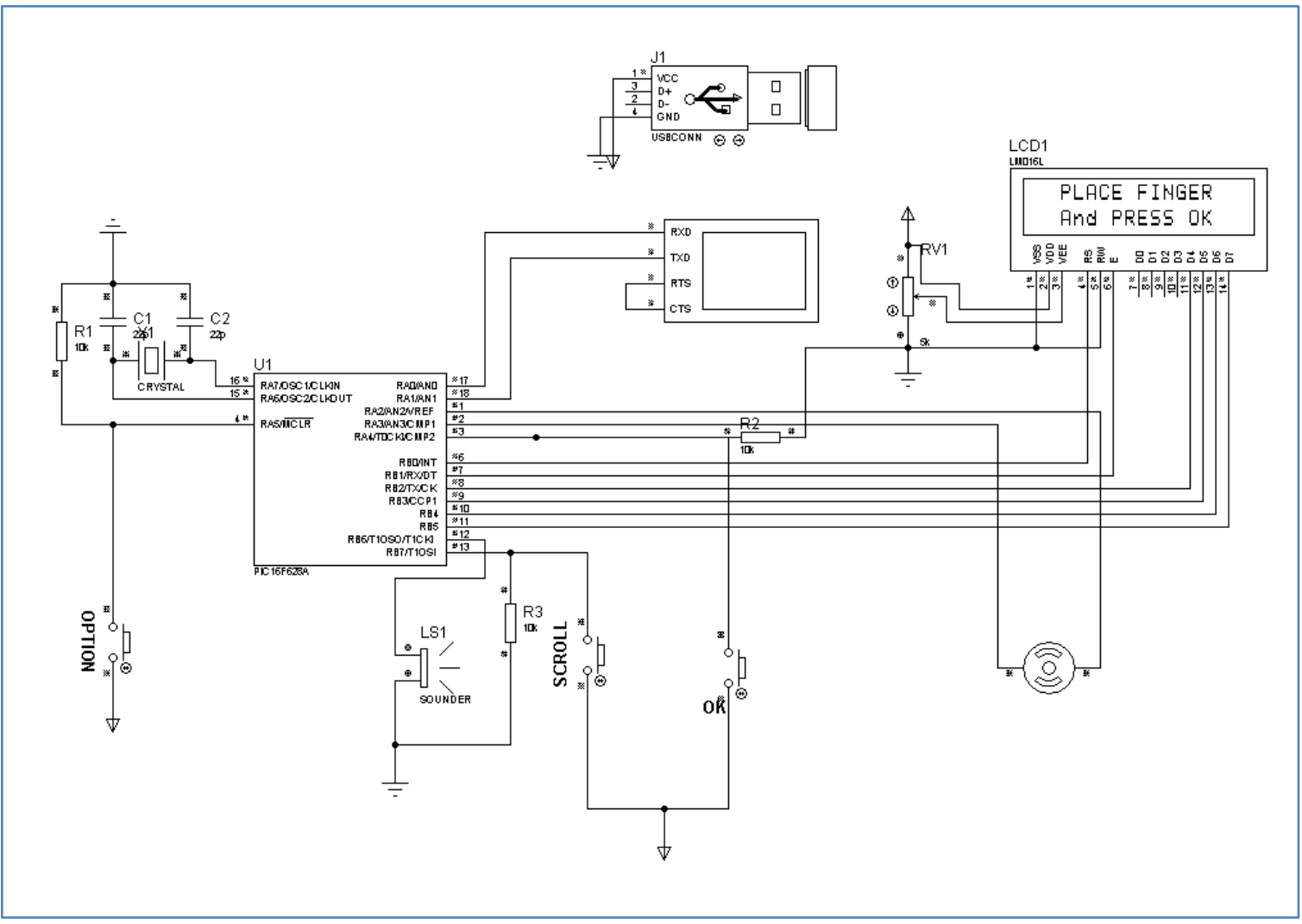

Figures 4.1c shows the simulation screen shot after registering finger print (default).

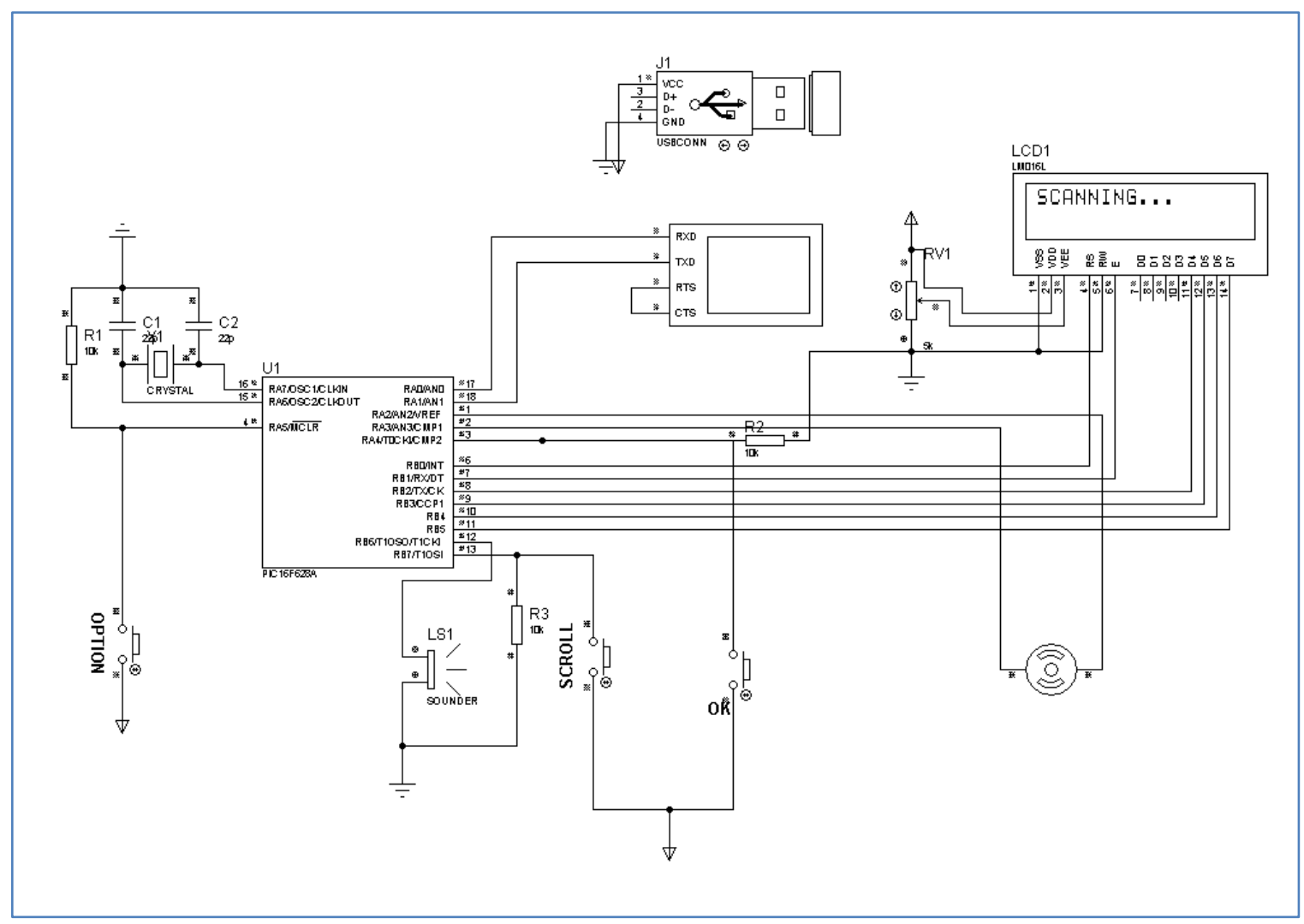

Figures 4.1d shows the simulation screen shot while scanning finger print. 


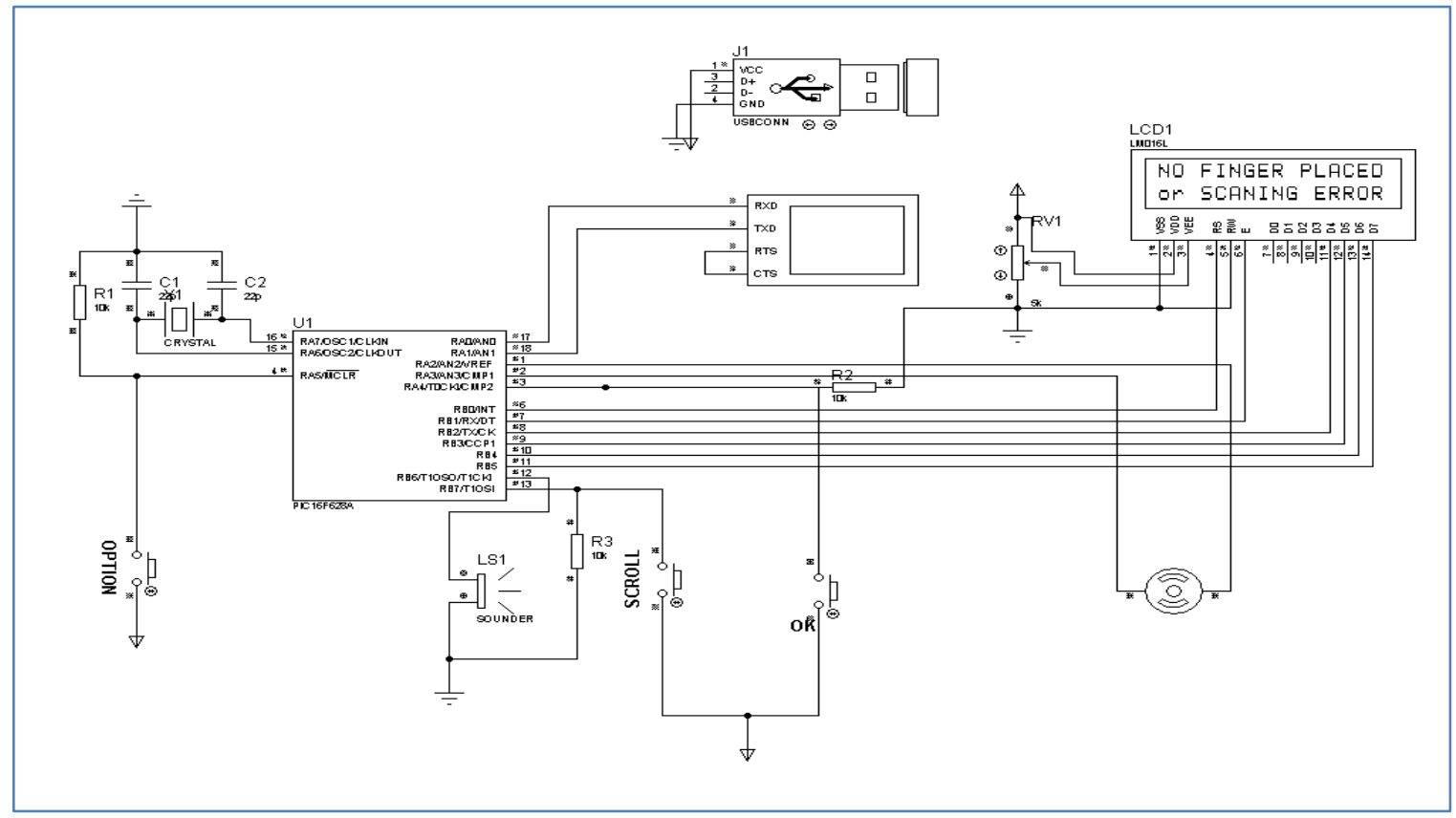

Figures 4.1e shows the simulation screen shot while error occurs at scanning finger print.

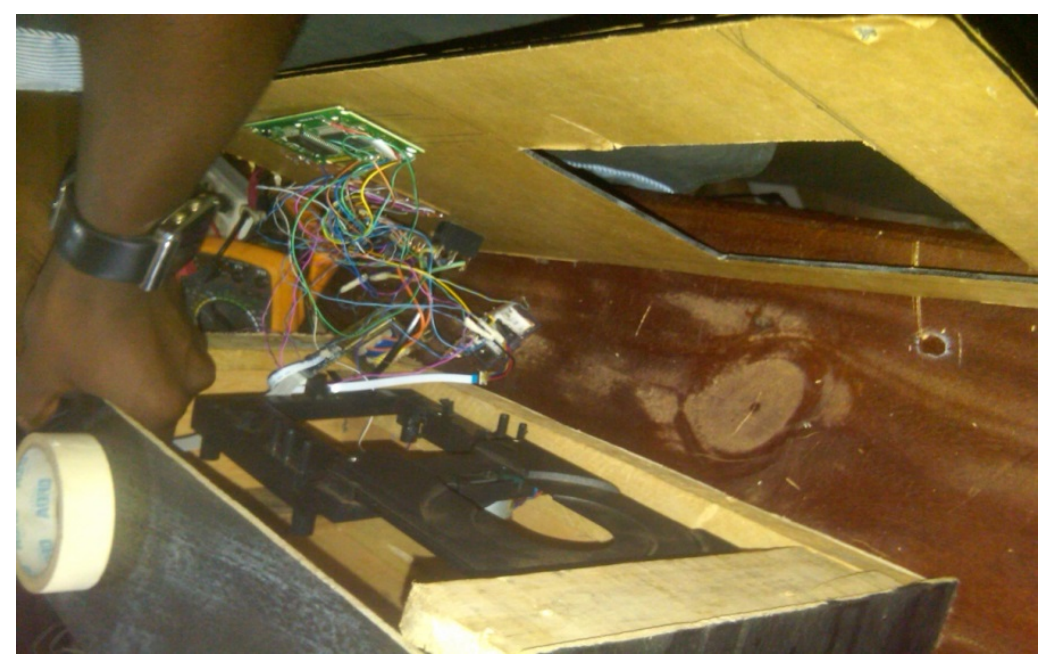

Figure 4.2 Picture of the door system before it was enclosed

\subsection{Soldering}

The various circuits and stages of this project were soldered in tandem to meet desired workability of the project. The power supply stage was first soldered before the microcontroller, digital (LCD) display and finger print reader module stages were done. The soldering of the project was done on Vero- board, and was soldered on two small sized Vero boards. The first Vero board contains the power supply stage, the microcontroller stage with the finger print reader module interface stage; the second Vero board has the transistor switching for driving the motor used to automate the door. 
Figure 4.3 below shows the soldering and component arrangement on the various Vero boards.

Vero-board 1 has the power supply stage which includes the rectification stage, filtering stage and the voltage regulation stage; and the microcontroller stage.

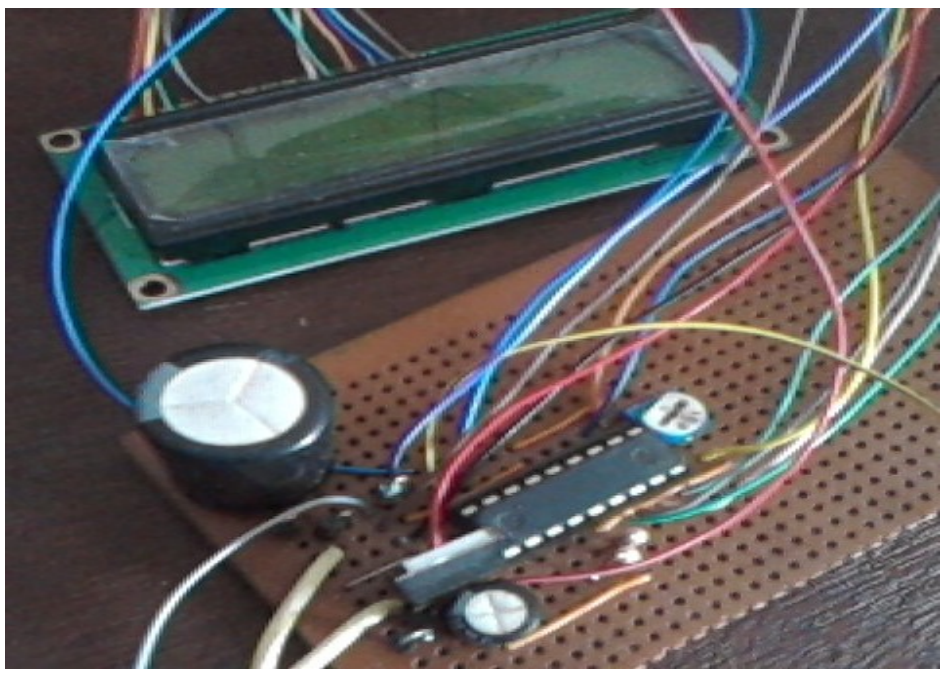

Figure 4.3 components layout on Vero-board.

\subsection{Testing}

Stage by stage testing was done according to the block representation on the breadboard, before soldering of circuit commenced on Vero board.

The process of testing and implementation involved the use of some test and measuring equipment stated below.

1. Bench Power Supply: This was used to supply voltage to the various stages of the circuit during the breadboard test before the power supply in the project was soldered. Also during the soldering of the project the power supply was still used to test various stages before they were finally soldered.

2. Oscilloscope: The oscilloscope was used to observe the ripples in the power supply waveform and to ensure that all waveforms were correct and their frequencies accurate. The waveform of the oscillation of the crystal oscillator used was monitor to ensure proper oscillation at $4 \mathrm{MHz}$.

3. Digital Multi-meter: The digital multi-meter basically measures voltage, resistance, continuity, current, frequency, temperature and transistor $h_{f e}$. The process of implementation of the design on the board required the measurement of parameters like, voltage, continuity, current and resistance values of the components and in some cases frequency measurement. The digital multimeter was used to check the output of the voltage regulators used in this project. 


\section{CONCLUSION}

The design is achieved by interfacing a SN-FPR-UART Finger reader with the PIC16F648A Microcontroller, it is the brain box that controls the whole secured door system. An LCD status display is employed to show the operating status of the system. A door movement mechanism is used in the design to make the automated door system move in clockwise and anti-clockwise direction, then finger print input stage was implemented using the SN-FPR-UART. It is a compact fingerprint reader module which is specially designed for project development because of its robustness and the following outstanding features. The development guarantees security for illegal intrusion of any entity to the room, the mechanism can be implemented in a broader sense on a door where there is restriction of access.

\section{REFERENCES}

[1] Cappellir. (2003) "Handbook of Fingerprint Recognition". Chapter synthetic fingerprint generation. Springer, New York.

[2] Cappelli R., M. Ferrara, and D. Maltoni (2006). "The Quality of Fingerprint Scanners and Its Impact on the Accuracy of Fingerprint Recognition Algorithms". In Processing of Multimedia Content Representation, Classification and Security (MRCS2006), pp $10\{16$.

[3] Sukthanker, G (1999), "Face Recognition: A critical look at Biologically-Inspired Approaches", Vol. 2, pp 45-48.

[4] Tang Homan and Sunny (2001) "Face Recognltion Review" Term paper, Department Computer Science and Engineering, Chinese University of Hong Kong, Shatin.

[5] Valetin D, Abdi H, O' Toole A.J. and Cottrell G.W. (1994), “Connectionist models of Face Processing: A survey", Pattern recognition, Vol.27, No.9, pp1209-1230.

[6] KarlJ.Astrom,Bjorn Wittenmark (1996) Computer Controlled Systems :Theory and Design.pp 23-34

[7] Simon Cole, Suspect Identities: A history of fingerprinting and criminal identification (Cambridge, Mass.: Harvard University Press, 2001), pages 60-61.

[8] Anil. K Jain, Arum Ross, and Prabhakar. S "Fingerprint Matching using Minutiae and Texture features", proc. International conference on image processing (ICIP),pp 282-285, Greece, October 7-10, 2003 